\title{
Schwinger's Dynamical Casimir Effect: Bulk Energy Contribution
}

\author{
C. E. Carlson*, C. Molina-París ${ }^{+}$, J. Pérez-Mercader ${ }^{++}$, and Matt Visser ${ }^{+++}$ \\ *Physics Department, College of William and Mary, Williamsburg, Virginia 23187 \\ + Theoretical Division, Los Alamos National Laboratory, Los Alamos, New Mexico 87545 \\ ${ }^{++}$Laboratorio de Astrofísica Espacial y Física Fundamental, Apartado 50727, 28080 Madrid \\ ${ }^{++}$Physics Department, Washington University, St. Louis, Missouri 63130-4899
}

(23 September 1996)

\begin{abstract}
Schwinger's Dynamical Casimir Effect is one of several candidate explanations for sonoluminescence. Recently, several papers have claimed that Schwinger's estimate of the Casimir energy involved is grossly inaccurate. In this letter, we show that these calculations omit the crucial volume term. When the missing term is correctly included one finds full agreement with Schwinger's result for the Dynamical Casimir Effect. We have nothing new to say about sonoluminescence itself except to affirm that the Casimir effect is energetically adequate as a candidate explanation.
\end{abstract}

\section{INTRODUCTION}

Several years ago, Schwinger wrote a series of papers [1,2, 3] wherein he calculated the Casimir energy released in the collapse of a spherically symmetric bubble or cavity. Using general arguments, he showed that the effect was mostly a volume effect, and derived a simple and elegant formula for the energy release involved in the collapse. He found that (for each polarization state) the "dielectric energy, relative to the zero energy of the vacuum, [is given] by

$$
E=-V \int \frac{d^{3} \vec{k}}{(2 \pi)^{3}} \frac{1}{2}[\hbar c] k\left(1-\frac{1}{\sqrt{\epsilon}}\right) .
$$

So the Casimir energy of a uniform dielectric is negative". From the above one finds that a dielectric slab with a spherical vacuum cavity of radius $R$ has a higher Casimir energy than the same slab of material with the cavity re-filled with dielectric. Introducing a wavenumber cutoff $K$ into the previous expression, 1 and summing over polarization states, shows that the Casimir energy of a cavity in a dielectric, relative to pure dielectric, is

$$
\begin{aligned}
E_{\text {cavity }} & =+2 \frac{4 \pi}{3} R^{3} \int_{0}^{K} \frac{4 \pi k^{2} d k}{(2 \pi)^{3}} \frac{1}{2} \hbar c k\left(1-\frac{1}{\sqrt{\epsilon}}\right) \\
& =+\frac{1}{6 \pi} \hbar c R^{3} K^{4}\left(1-\frac{1}{\sqrt{\epsilon}}\right) .
\end{aligned}
$$

In general, this volume term will be the dominant contribution.

\footnotetext{
* For sonoluminescence, this wave-number cutoff can be related to the wavelength of the electromagnetic radiation emitted in the collapse of the bubble. For generic dielectrics, this wave-number cutoff is related to the high-wave-number asymptotic behaviour of the dispersion relation.
}

In view of the elegance and simplicity of this result, it is natural to ask whether it can also be derived by more traditional quantum field theoretic means. Indeed, the existence of such a volume contribution is easy to verify on general physical grounds:

(1) One can view Schwinger's result in elementary terms as simply the difference in zero-point-energies, obtained by integrating the difference if photon dispersion relations over the density of statest

$$
E_{\text {cavity }}=+2 V \int \frac{d^{3} \vec{k}}{(2 \pi)^{3}} \frac{1}{2} \hbar[c k-\omega(k)]+\cdots
$$

At low wave-numbers, we know that the dispersion relation for a dielectric is simply summarized by the zerofrequency refractive index $n$. That is

$$
\omega(k) \rightarrow c k / n \quad \text { as } \quad k \rightarrow 0 .
$$

On the other hand, at high enough wave-numbers, the photons propagate freely through the dielectric: They are then simply free photons travelling through the empty vacuum between individual atoms. Thus

$$
\omega(k) \rightarrow c k \quad \text { as } \quad k \rightarrow \infty .
$$

From the above we know that the integrand must go to zero at large wave-number. In fact for any real dielectric the integrand must go to zero sufficiently rapidly to make the integral converge, since after all we are talking about a real physical difference in energies.

To actually calculate this energy difference one requires a suitable physical model for $\omega(k)$. Schwinger's calculation [1], is equivalent to picking the particularly simple model

\footnotetext{
${ }^{\dagger}$ The dots denote finite-volume corrections. We shall develop this density-of-states point of view more fully in a separate publication.
} 


$$
\omega(k)=\frac{c k}{n} \Theta(K-k)+c k \Theta(k-K) .
$$

Here $\Theta(x)$ is the Heaviside step function, and $K$ is a wave-number which characterizes the transition from dielectric-like behaviour to vacuum-like behaviour. Note that the cutoff $K$ describes an actual physical situation: It is a surrogate for all of the complicated physics that would be required to make a detailed model for the dielectric to vacuum transition.

(2) We also know that the quantum action in $3+1$ dimensions generically contains divergences which range from quartic to logarithmic, in addition to finite contributions. As is well known, this "cosmological constant" contribution (the quartic divergence) will not vanish unless the theory has very special symmetries (for example - supersymmetry). Thus energy densities that go as (cutoff $)^{4}$ are generic in $(3+1)$ dimensions.

(3) Alternatively, one could perform an explicit quantum field theoretic calculation of the Casimir energy in some model problem and thereby verify Schwinger's result. A step in this direction has been provided by Milton et al. [4,5,5], who attempted to compute the Casimir energy associated with a spherical cavity of radius $R$, dielectric constant $\epsilon_{1}$, and permeability $\mu_{1}$, embedded in an infinite medium with dielectric constant $\epsilon_{2}$ and permeability $\mu_{2}$. They found that the dominant term is not the volume term but a surface term which is proportional to $R^{2} K^{3}\left(\epsilon_{1}-\epsilon_{2}\right)^{2}$. We, however, have re-analysed these calculations and do find a volume term which dominates except for very small bubbles.

We have performed the calculation of the Casimir energy in two different and complementary ways:

(a) We have taken the formalism of Milton et al. [4, 5, 6] and applied it directly to an ab initio calculation of the Casimir energy. We compute the energy difference between the following configurations: (Case I) an otherwise uniform medium with dielectric constant $\epsilon_{2}$ and permeability $\mu_{2}$, containing a spherical cavity of radius $R$ with dielectric constant $\epsilon_{1}$ and permeability $\mu_{1}$, and (Case II) a completely uniform medium with dielectric constant $\epsilon_{2}$ and permeability $\mu_{2}$. This energy difference is given as a sum over a series of integrals involving Ricatti-Bessel functions. Some of the sums can be evaluated explicitly while others can only be evaluated by using an asymptotic analysis of the type used by Milton et al. We verify the existence of both volume and sub-dominant surface contributions.

(b) We have analyzed the extant calculations to see exactly where they differ from the present calculation. We find that the subtraction scheme they use to calculate the Casimir energy does not correspond to the physical situation in question. We isolate the difference in energy between these calculations and the correct one. We will explicitly show that this difference is proportional to volume.

\section{PHYSICAL DESCRIPTION OF THE CALCULATION}

Milton et al. [4,5,5] explicitly calculated the electromagnetic Green functions for a dielectric ball embedded in an infinite space of (different) dielectric material, and then attempted to calculate the Casimir energy by explicitly integrating these Green functions over "all space". Note that an important limitation of any such calculation is that any attempt at explicitly calculating Green functions must be restricted to systems of extremely high symmetry - such as half-spaces, slabs, or balls. The basic strategy is to take the classical expression for the energy

$$
E=\frac{1}{2} \int_{\text {Geometry }}\left[\epsilon \vec{E}^{2}+\frac{1}{\mu} \vec{B}^{2}\right] d^{3} x,
$$

promote the electric and magnetic fields to be operator quantities, and then calculate the vacuum expectation value

$$
\begin{aligned}
& E=\frac{1}{2} \int_{\text {Geometry }} {[\epsilon\langle\vec{E}(0, x) \cdot \vec{E}(0, x)\rangle} \\
&\left.+\frac{1}{\mu}\langle\vec{B}(0, x) \cdot \vec{B}(0, x)\rangle\right] d^{3} x .
\end{aligned}
$$

The geometry is incorporated in the calculation both via the limits of integration and via the boundary conditions satisfied by the fields. Since these two-point functions are of course divergent, they must be rendered finite by some regularization prescription. Milton et al. use pointsplitting in the time direction:

$$
\begin{aligned}
E(\tau)=\frac{1}{2} \int_{\text {Geometry }}[\epsilon\langle\vec{E}(\tau, x) \cdot \vec{E}(0, x)\rangle \\
\left.+\frac{1}{\mu}\langle\vec{B}(\tau, x) \cdot \vec{B}(0, x)\rangle\right] d^{3} x .
\end{aligned}
$$

All of the technical aspects of the analysis then focus on the calculation of these two-point correlation functions (Green functions) by explicitly solving for the TE and TM modes appropriate for a spherical ball with dielectric boundary conditions; and then explicitly writing down the Green functions as a sum over suitable combinations of Ricatti-Bessel functions and vector spherical harmonics. To avoid unnecessary notational complications, we schematically rewrite the above as

$$
E(\tau)=\frac{1}{2} \int_{\text {Geometry }} G_{[\epsilon, \mu]}(\tau, x ; 0, x) d^{3} x,
$$

where $G_{[\epsilon, \mu]}\left(t, x ; t^{\prime}, x^{\prime}\right)$ is simply shorthand for the linear combination of Green functions appearing above.

We may calculate these Green functions for three different geometriest:

\footnotetext{
${ }^{\ddagger}$ Notice that in Milton et al. the dielectric properties of these media are taken to be frequency independent, the cutoff being put in "by hand" via time-splitting.
} 
Case I: A dielectric ball of dielectric constant $\epsilon_{1}$, permeability $\mu_{1}$, and radius $R$ embedded in a infinite dielectric of different dielectric constant $\epsilon_{2}$ and permeability $\mu_{2}$. (In applications to sonoluminescence, think of this as an air bubble of radius $R$ in water.)

Case II: A completely homogeneous space completely filled with dielectric $\left(\epsilon_{2}, \mu_{2}\right)$. (In applications to sonoluminescence, think of this as pure water.)

Case III: A completely homogeneous space completely filled with dielectric $\left(\epsilon_{1}, \mu_{1}\right)$. (In applications to sonoluminescence, think of this as pure air.)

We are in complete agreement with the extant calculations and results for these three individual Green functions - where we disagree, as will be shown, is in the way that these three Green functions are inserted into the computation for the Casimir energy.

Milton et al. calculate an "energy difference", which we will call $E_{\text {surface }}$, and which they define as

$$
E_{\text {surface }}=\frac{1}{2}\left\{\int_{\text {all r }} G_{I}-\int_{r>R} G_{I I}-\int_{r<R} G_{I I I}\right\} .
$$

The computation of this quantity in [田, 厉] is mathematically correct. An asymptotic analysis shows that this expression is indeed proportional to the surface areaplus even higher-order terms. However, the energy calculated from the above expression does not correspond to the energy of a physically realizable situation.

The physically correct quantity to compute is [1]

$$
E_{\text {Casimir }}=\frac{1}{2}\left\{\int_{\text {all r }} G_{I}-\int_{\text {all r }} G_{I I}\right\} .
$$

Observe that this quantity is simply the difference in energy between two real physical situations: (Case I) having the dielectric ball present and (Case II) replacing the dielectric ball by the surrounding medium. This is exactly the quantity that Schwinger calculates in reference []] to describe the Casimir enegy released in the collapse of the bubble: it is the energy released in evolving from bubble to no-bubble. The difference between the two calculations is

$$
\Delta E=E_{\text {Casimir }}-E_{\text {surface }}=\frac{1}{2} \int_{r<R}\left\{G_{I I I}-G_{I I}\right\} .
$$

This difference is easily seen to be proportional to the volume: remember that $G_{I I I}$ and $G_{I I}$ are Green functions corresponding to two spaces that are completely filled with homogeneous dielectrics - therefore they are each individually translation invariant. (When one expresses these Green functions in terms of spherical polar coordinates this is not obvious.) This observation permits one to pull the Green functions outside the integral, so that

$$
\Delta E=\frac{1}{2} V\left\{G_{I I I}(\tau, 0 ; 0,0)-G_{I I}(\tau, 0 ; 0,0)\right\},
$$

where $V$ is the volume of the ball of radius $R$. We shall now show that this term is in fact exactly in conformity with Schwinger's result.

\section{III. “AB INITIO" CALCULATION}

\section{A. The energy density}

We now calculate the Casimir energy for the geometrical configuration previously described. We use techniques developed by Milton et al., but use, as Schwinger did, a wave number cutoff and shall present the calculation in as much detail as space permits. We defer many technical details to a forthcoming publication.

For each individual geometry, the energy density $T^{t t}$ can be evaluated by using the dyadic Green function formalism [4, 5.6. (Henceforth we use natural units.) For Case I one finds

$$
T_{I}^{t t}(r)=\operatorname{Re}\left[\frac{-i}{8 \pi} \int_{-\infty}^{+\infty} \frac{d \omega}{2 \pi} e^{-i \omega \tau} X_{I}(k, r)\right],
$$

with identical expressions holding for the other geometries. Here the $\omega$-integral arises from the time-splitting regularization, while we have used the notation $k=|\omega| n$ with $n$ the appropriate position-dependent refractive index ( $n_{1}$ inside the dielectric sphere, $n_{2}$ outside), and have defined the quantity $X_{I}(k, r)$ by

$$
\begin{aligned}
X_{I}(k, r) \equiv & \sum_{\ell=1}^{\infty}(2 \ell+1)\left\{\left[k_{I}^{2}+\frac{\ell(\ell+1)}{r^{2}}\right] F_{\ell}^{I}(k ; r, r)\right. \\
& \left.+\left.\frac{1}{r^{2}} \frac{\partial}{\partial r_{1}} r_{1} \frac{\partial}{\partial r_{2}} r_{2}\left[F_{\ell}^{I}\left(k ; r_{1}, r_{2}\right)\right]\right|_{r_{1}=r_{2}=r}\right\} \\
& +\left[\left(F_{\ell}^{I}\right) \rightarrow\left(G_{\ell}^{I}\right)\right] .
\end{aligned}
$$

The functions $F_{\ell}\left(r, r^{\prime}\right)$ and $G_{\ell}\left(r, r^{\prime}\right)$ are the Green functions for the electrical and magnetic fields in the appropriate geometry, and are given below. Similar results, with appropriate substitutions for the momenta, hold when one makes reference to Cases II and III. Note that when making the substitutions $(I) \rightarrow(I I)$ or $(I) \rightarrow(I I I)$, one should also change the refractive index that implicitly appears in the factor $k$. In addition it should be borne in mind that $k_{I}$ is a function of position: $k_{I}=n_{1}|\omega|=k_{I I I}$ inside the dielectric sphere, whereas $k_{I}=n_{2}|\omega|=k_{I I}$ outside the dielectric sphere.

Because $F_{\ell}$ and $G_{\ell}$ depend only on the absolute value of $\omega$ we can write the energy density as

$$
\begin{aligned}
T_{I}^{t t}(r) & =\operatorname{Re}\left[\frac{-i}{8 \pi} \int_{0}^{\infty} \frac{d \omega}{2 \pi}\left[e^{-i \omega \tau}+e^{+i \omega \tau}\right] X_{I}(k, r)\right] \\
& =\operatorname{Re}\left[\frac{-i}{4 \pi} \int_{0}^{\infty} \frac{d \omega}{2 \pi} \cos (\omega \tau) X_{I}(k, r)\right]
\end{aligned}
$$




$$
=\frac{1}{4 \pi} \int_{0}^{\infty} \frac{d \omega}{2 \pi} \cos (\omega \tau) \operatorname{Im}\left[X_{I}(k, r)\right] .
$$

The Casimir energy, Eq. (12) is obtained by taking the difference in energy densities and integrating over all space while paying attention to the appropriate index of refraction for each region of space. Thus

$$
\begin{aligned}
E_{\text {Casimir }}= & \int_{0}^{\infty} r^{2} d r \int_{0}^{\infty} \frac{d \omega}{2 \pi} \cos (\omega \tau) \\
& \times \operatorname{Im}\left[X_{I}(k, r)-X_{I I}(k, r)\right] .
\end{aligned}
$$

This expression for the Casimir energy is completely equivalent to equation (41) of [5], and equation (4.2b) of [6] and is also closely related to equations (30a) and (30b) of [4]. Note that extant calculations use the same time-splitting parameter for the two different media - the physics behind this choice is far from clear, and we shall return to this point in a future publication.

It is now clear how one should modify these expressions to replace time-splitting regularization by a wave-number cutoff. For generality we can take an arbitrary wavenumber cutoff described by some smooth real function $f(k)$ which goes to zero as $k \rightarrow \infty$ and simply write

$$
\begin{aligned}
E_{\text {Casimir }}= & \int_{0}^{\infty} r^{2} d r \int_{0}^{\infty} \frac{d \omega}{2 \pi} \\
& \times \operatorname{Im}\left[f\left(k_{I}\right) X_{I}(k, r)-f\left(k_{I I}\right) X_{I I}(k, r)\right] .
\end{aligned}
$$

With due caution, the relevant Green functions can be read off from [4, 5,6$]$.

\section{B. The Green functions}

In evaluating the Green functions one must be careful to correctly incorporate the boundary conditions appropriate to the geometry and the physics. This means that they must satisfy appropriate continuity conditions derived from Maxwell's equations. That is

$$
\vec{E}_{\perp}, \quad \epsilon \vec{E}_{r}, \quad \frac{1}{\mu} \vec{B}_{\perp}, \quad \text { and } \quad \vec{B}_{r}
$$

must be continuous. In terms of $F_{\ell}$ and $G_{\ell}$, one sees that

$$
\mu F_{\ell}, \quad G_{\ell}, \quad \frac{\partial}{\partial r} r F_{\ell}, \quad \text { and } \quad \frac{1}{\epsilon} \frac{\partial}{\partial r} r G_{\ell},
$$

must be continuous. The Green functions are

\section{Case I:}

For $r_{1}, r_{2}<R$ :

$$
\begin{aligned}
F_{\ell}^{I}, G_{\ell}^{I}\left(r_{1}, r_{2}\right)= & i k_{I I I} j_{\ell}\left(k_{I I I} r_{<}\right) \\
& \times\left[h_{\ell}\left(k_{I I I} r_{>}\right)-A_{F, G}^{\ell} j_{\ell}\left(k_{I I I} r_{>}\right)\right] .
\end{aligned}
$$

For $r_{1}, r_{2}>R$ :

$$
\begin{aligned}
F_{\ell}^{I}, G_{\ell}^{I}\left(r_{1}, r_{2}\right)= & i k_{I I} h_{\ell}\left(k_{I I} r_{>}\right) \\
& \times\left[j_{\ell}\left(k_{I I} r_{<}\right)-B_{F, G}^{\ell} h_{\ell}\left(k_{I I} r_{<}\right)\right] .
\end{aligned}
$$

The function $j_{\ell}(x)$ is the spherical Bessel function of order $\ell$ and $h_{\ell}(x) \equiv h_{\ell}^{(1)}(x)$ is the spherical Hankel function of the first kind. See equations (12a) and (12b) of [4, equation (16) of [5], or equation (2.13) of [6]. The quantities $A_{F, G}^{\ell}$ and $B_{F, G}^{\ell}$ are those given in 4,5$]$.

Case II:

For all $r_{1}, r_{2}$ :

$$
F_{\ell}^{I I}, G_{\ell}^{I I}\left(r_{1}, r_{2}\right)=i k_{I I} j_{\ell}\left(k_{I I} r_{<}\right) h_{\ell}\left(k_{I I} r_{>}\right) .
$$

\section{Case III:}

For all $r_{1}, r_{2}$ :

$$
F_{\ell}^{I I I}, G_{\ell}^{I I I}\left(r_{1}, r_{2}\right)=i k_{I I I} j_{\ell}\left(k_{I I I} r_{<}\right) h_{\ell}\left(k_{I I I} r_{>}\right) .
$$

We are now ready to explicitly compute the Casimir energy. In passing we remark that the object $F_{\ell}^{(0)}$ defined in equation (29) of [4], equation (35) of [5], and equation (3.7) of [6], which is essential for those calculations, is not a Green function of any differential operator. Specifically, $F_{\ell}^{(0)}$ does not satisfy the dielectric boundary conditions. It is not even continuous, and is merely a potpourri of two different Green functions which does not have any particular physical relevance.

\section{The Casimir energy}

We calculate $E_{\text {Casimir }}$ using equation 19), [equivalently (18)] together with equations (22 24). When evaluating the imaginary parts of $X$ it is convenient to introduce the Ricatti-Bessel functions $s_{\ell}(x)=x j_{\ell}(x)$ and $e_{\ell}(x)=x h_{\ell}(x)$. One also needs the identity

$$
\frac{\ell(\ell+1)}{x^{2}} s_{\ell}(x)=s_{\ell}^{\prime \prime}(x)+s_{\ell}(x)
$$

together with an identical equation which holds for $e_{\ell}(x)$. After some rearrangement (technical details are suppressed and will be relegated to a more detailed forthcoming publication) we find that inside the dielectric sphere

$$
\begin{aligned}
& \operatorname{Im}\left\{X_{I}(k, r)\right\}_{i n} \\
&= 2 \frac{k_{I I I}}{r^{2}} \sum_{\ell=1}^{\infty}(2 \ell+1) \\
& \times\left.\left\{2\left[s_{\ell}(x)\right]^{2}+\left[s_{\ell}^{\prime}(x)\right]^{2}+s_{\ell}(x) s_{\ell}^{\prime \prime}(x)\right\}\right|_{k_{I I I} r} \\
&- \frac{k_{I I I}}{r^{2}} \sum_{\ell=1}^{\infty}(2 \ell+1) \operatorname{Re}\left\{A_{F}^{\ell}+A_{G}^{\ell}\right\} \\
& \times\left.\left\{2\left[s_{\ell}(x)\right]^{2}+\left[s_{\ell}^{\prime}(x)\right]^{2}+s_{\ell}(x) s_{\ell}^{\prime \prime}(x)\right\}\right|_{k_{I I I} r}
\end{aligned}
$$

A remarkable Ricatti-Bessel function identity permits us to perform the first sum over $\ell$ exactly. Using

$$
\sum_{\ell=1}^{\infty}(2 \ell+1)\left[2 s_{\ell}(x)^{2}+s_{\ell}^{\prime}(x)^{2}+s_{\ell}(x) s_{\ell}^{\prime \prime}(x)\right]=2 x^{2},
$$


one obtains that

$$
\begin{aligned}
& \operatorname{Im}\left\{X_{I}(k, r)\right\}_{i n}=4 k_{I I I}^{3} \\
& -\frac{k_{I I I}}{r^{2}} \sum_{\ell=1}^{\infty}(2 \ell+1) \operatorname{Re}\left\{A_{F}^{\ell}+A_{G}^{\ell}\right\} \\
& \quad \times\left.\left\{2\left[s_{\ell}(x)\right]^{2}+\left[s_{\ell}^{\prime}(x)\right]^{2}+s_{\ell}(x) s_{\ell}^{\prime \prime}(x)\right\}\right|_{k_{I I I} r} .
\end{aligned}
$$

Note that for the $A_{F, G^{\ell}}$-terms we cannot explicitly perform the $\ell$ summation because of the complicated $\ell$ dependence of these coefficients $[5,6$.

It is very important to notice at this point that the $A_{F, G}^{\ell}$-terms in the above expressions are the only pieces retained in the currently extant calculations, the other terms unfortunately have been missed there due to the use of the wrong "Green functions".

Taking a cue from the above, the results for the region inside the bubble can be written (using self explanatory notation) as

$$
\operatorname{Im}\left\{X_{I}(k, r)\right\}_{\text {in }}=4 n_{1}^{3}|\omega|^{3}+Q_{\text {in }}^{\text {surface }}\left(k_{I I I}, r\right) .
$$

Turning to the region outside the dielectric sphere, one gets that

$$
\begin{aligned}
& \operatorname{Im}\left\{X_{I}(k, r)\right\}_{\text {out }} \\
&=2 \frac{k_{I I}}{r^{2}} \sum_{\ell=1}^{\infty}(2 \ell+1) \\
& \times\left.\left\{2\left[s_{\ell}(x)\right]^{2}+\left[s_{\ell}^{\prime}(x)\right]^{2}+s_{\ell}(x) s_{\ell}^{\prime \prime}(x)\right\}\right|_{k_{I I} r} \\
&- \frac{k_{I I}}{r^{2}} \sum_{\ell=1}^{\infty}(2 \ell+1) \operatorname{Re}\left[\left(B_{F}^{\ell}+B_{G}^{\ell}\right)\right. \\
&\left.\times\left\{2\left[e_{\ell}(x)\right]^{2}+\left[e_{\ell}^{\prime}(x)\right]^{2}+e_{\ell}(x) e_{\ell}^{\prime \prime}(x)\right\}\right]\left.\right|_{k_{I I I} r} \\
&= 4 k_{I I}^{3}-\frac{k_{I I}}{r^{2}} \sum_{\ell=1}^{\infty}(2 \ell+1) \operatorname{Re}\left[\left(B_{F}^{\ell}+B_{G}^{\ell}\right)\right. \\
&\left.\times\left\{2\left[e_{\ell}(x)\right]^{2}+\left[e_{\ell}^{\prime}(x)\right]^{2}+e_{\ell}(x) e_{\ell}^{\prime \prime}(x)\right\}\right]\left.\right|_{k_{I I I} r} .
\end{aligned}
$$

Again, in self-explanatory notation

$$
\operatorname{Im}\left\{X_{I}(k, r)\right\}_{\text {out }}=4 n_{2}^{3}|\omega|^{3}+Q_{\text {out }}^{\text {surface }}\left(k_{I I}, r\right) .
$$

For Case II one simply has

$$
\begin{aligned}
\operatorname{Im}\left\{X_{I I}(k, r)\right\}_{\text {all space }}=2 \frac{k_{I I}}{r^{2}} \sum_{\ell=1}^{\infty}(2 \ell+1) \\
\quad \times\left\{2\left[s_{\ell}(x)\right]^{2}+\left[s_{\ell}^{\prime}(x)\right]^{2}+s_{\ell}(x) s_{\ell}^{\prime \prime}(x)\right\}||_{k_{I I} r} \\
=4 k_{I I}^{3}=4 n_{2}^{3}|\omega|^{3} .
\end{aligned}
$$

Going back to the momentum-space regulated Casimir energy, equation (19), we obtain

$$
E_{\text {Casimir }}=\int_{0}^{R} r^{2} d r \int_{0}^{\infty} \frac{d \omega}{2 \pi} 4|\omega|^{3}
$$

$$
\begin{gathered}
\times\left[n_{1}^{3} f\left(n_{1}|\omega|\right)-n_{2}^{3} f\left(n_{2}|\omega|\right)\right] \\
+\int_{0}^{R} r^{2} d r \int_{0}^{\infty} \frac{d \omega}{2 \pi} f\left(n_{1}|\omega|\right) Q_{\text {in }}^{\text {surface }}(k, r) \\
+\int_{R}^{\infty} r^{2} d r \int_{0}^{\infty} \frac{d \omega}{2 \pi} f\left(n_{2}|\omega|\right) Q_{\text {out }}^{\text {surface }}(k, r) .
\end{gathered}
$$

The remaining integrals for the $n^{3}|\omega|^{3}$ term are trivial. Changing the integration variable to $k=n|\omega|$, and explicitly re-inserting the appropriate factors of $\hbar$ and $c$, we get

$$
\begin{aligned}
E_{\text {Casimir }} & =+2 V \int \frac{d^{3} \vec{k}}{(2 \pi)^{3}} \frac{1}{2} \hbar\left[\omega_{1}(k)-\omega_{2}(k)\right] f(k) \\
& +\int_{0}^{R} r^{2} d r \int_{0}^{\infty} \frac{d \omega}{2 \pi} \hbar f\left(n_{1}|\omega|\right) Q_{\text {in }}^{\text {surface }}(k, r) \\
& +\int_{R}^{\infty} r^{2} d r \int_{0}^{\infty} \frac{d \omega}{2 \pi} \hbar f\left(n_{2}|\omega|\right) Q_{\text {out }}^{\text {surface }}(k, r) .
\end{aligned}
$$

which is the central result of this paper.

This is explicitly of the form:

(Schwinger's volume term) + (surface term)

The "surface term" corresponds to $E_{\text {surface }}$ and is given by the two double integrals in the expression for $E_{\text {Casimir }}$ above. For time-splitting regularization and dilute dielectric media, these terms were explicitly shown by Milton et al. to be proportional to the surface area (plus even higher-order corrections). The first term is the volume term not present in some of the existing calculations. In fact, after approximating air by vacuum (setting $n_{1}=1$ ) and using Schwinger's momentum space cutoff, this integral is exactly equal to Schwinger's result [1].

\section{DISCUSSION}

The main result of this paper can be succinctly stated: in a dielectric medium of dielectric constant $n$ the Casimir energy of a cavity - the difference in zero point energies of a dielectric medium of refractive index $n$ with and without a vacuum cavity of volume $V$-is:

$$
E_{\text {Casimir }}=\frac{1}{8 \pi^{2}} V \hbar c K^{4}\left[1-\frac{1}{n}\right]+\cdots,
$$

with this volume term dominant if the scale of the bubble is larger than the cutoff wavelength $2 \pi / K$. This result is completely in agreement with Schwinger's calculation in [1], and Schwinger's argument is now buttressed by our explicit re-assessment of Milton et al.'s calculation for a spherical dielectric ball.

We close with what is perhaps a minor point that we nevertheless feel should be made explicit: the volume 
contribution to the Casimir energy is always there, and is always physical, but it is sometimes safe to neglect it.

For example, a situation equally physical as the one we have considered here is the following: suppose one is provided with a fixed number of dielectric bodies of fixed shape (in particular, of fixed volume), and suppose that one simply wishes to move the bodies around in space with respect to each other. Then the bulk volume contributions to the Casimir energy, while still present, are constants independent of the relative physical location of the dielectric bodies, and so merely provide a constant offset to the total Casimir energy. If all we are interested in is the energy differences between different spatial configurations of the same bodies then the various volume contributions can be quietly neglected.

On the other hand, the volume contribution is of critical importance whenever one wants to calculate the energy difference between an inhomogeneous dielectric and a homogeneous dielectric wherein the irregularities have been filled in. This is, precisely, the physical situation in the case of bubble collapse in a dielectric medium.

\section{ACKNOWLEDGMENTS}

This work was supported in part by the U.S. Department of Energy, the U.S. National Science Foundation, by the Spanish Ministry of Education and Science and the Spanish Ministry of Defense. Part of this work was carried out at the Laboratory for Space Astrophysics and Fundamental Physics (LAEFF, Madrid), and C.E.C., C.M-P., and M.V. wish to gratefully acknowledge the hospitality shown. Part of this work was carried out at Los Alamos National Laboratory, and J.P-M. wishes to gratefully acknowledge the hospitality shown to him there.

[1] J. Schwinger, Proc. Nat. Acad. Sci. 90, 2105-2106 (1993).

[2] J. Schwinger, Proc. Nat. Acad. Sci. 90, 4505-4507 (1993).

[3] J. Schwinger, Proc. Nat. Acad. Sci. 90, 7285-7287 (1993).

[4] K. Milton, Annals of Physics (NY), 127, 49-61 (1980).

[5] K. Milton, Casimir energy for a spherical cavity in a dielectric: toward a model for Sonoluminescence?, in Quantum field theory under the influence of external conditions, edited by M. Bordag, (Tuebner Verlagsgesellschaft, Stuttgart, 1996), pages 13-23. See also hep-th/9510091. Warning: the equation numbers (though not the text) differ between the published version and the hep-th version-all equation numbers after (32) are incremented by 1 in the hep-th version.

[6] K. Milton and J. Ng, Casimir energy for a spherical cavity in a dielectric: Applications to Sonoluminescence, hepth/9607186. 